\title{
Análise fitoquímica, potencial antioxidante e toxicidade do extrato bruto etanólico e das frações da espécie Senecio westermanii Dusén frente à Artemia salina
}

\author{
MERINO, F.J.Z. ${ }^{*}$; OLIVEIRA, V.B. ${ }^{\prime}$; PAULA, C.S. ${ }^{2}$; CANSIAN, F.C.2; SOUZA, A.M.2; ZUCHETTO, M. ${ }^{2}$; HIROTA, \\ B.C.K.2; DUARTE, A.F.S.'; KULIK, J.D.'; MIGUEL, M.D.2; MIGUEL, O.G. ${ }^{1}$ \\ 'Universidade Federal do Paraná, Departamento de Farmácia, Programa de Pós Graduação em Ciências \\ Farmacêuticas, Laboratório de Fitoquímica. Av. Pref. Lothário Meissner, 632 - Jardim Botânico, CEP: 80210-170, \\ Curitiba, PR, Brasil; 2Universidade Federal do Paraná, Departamento de Farmácia, Programa de Pós Graduação \\ em Ciências Farmacêuticas, Laboratório de Farmacotécnica. Av. Pref. Lothário Meissner, 632 - Jardim Botânico, \\ CEP: 80210-170, Curitiba, PR, Brasil. *Autor para correspondência: esecocio@yahoo.com.br
}

RESUMO: A espécie Senecio westermanii Dusén pertencente à família Asteraceae é planta endêmica e nativa do Brasil encontrada na região da Floresta Atlântica, nos estados do Paraná e São Paulo. O objetivo deste trabalho foi avaliar a composição fitoquímica do extrato bruto etanólico e frações das partes aéreas (folha e caule) de $S$. westermanii utilizando-se a análise fitoquímica qualitativa e cromatografia líquida de alta eficiência com detector de arranjo de diodos (CLAE-DAD), avaliar in vitro a toxicidade preliminar utilizando Artemia salina e o potencial antioxidante. $\mathrm{O}$ estudo fitoquímico qualitativo revelou a presença de alcaloides, flavonoides, iridoides, esteroides/triterpenos, heterosídeos saponínicos e aminogrupos. Através da análise realizada por CLAE-DAD obteve-se o fingerprint característico de cada amostra. No ensaio frente à $A$. salina houve ausência de toxicidade das amostras, o resultado da $\mathrm{DL}_{50}$ para todas as amostras foi superior a $1000 \mu \mathrm{g} / \mathrm{mL}$. Todas as amostras apresentaram atividade antioxidante pela redução do complexo fosfomolibdênio, com destaque para a fração clorofórmio que apresentou atividade antioxidante de $92,51 \%$ em relação ao padrão rutina. Com relação ao ensaio de redução do radical DPPH•, a fração acetato de etila apresentou $\mathrm{IC}_{50}$ de $26,98 \mu \mathrm{g} /$ $\mathrm{mL}$. Pelo ensaio do ácido tiobarbitúrico (TBARS) a fração hexano apresentou o melhor índice antioxidante em relação ao padrão rutina. Os resultados obtidos demonstram evidências de que a espécie é fonte potencial de antioxidantes naturais, estimulando assim novos estudos que viabilizam sua utilização no tratamento de patologias associadas aos radicais livres. Além disso, a espécie não apresentou atividade tóxica preliminar, assegurando sua aplicabilidade.

Palavras-chave: Senecio; Análise fitoquímica; Artemia salina; Antioxidante; CLAE-DAD

\begin{abstract}
Phytochemical analysis, antioxidant potential and toxicity of crude ethanol extract and fractions of the species Senecio westermanii Dusén against Artemia salina. The species Senecio westermanii Dusén, which belongs to the Asteraceae family, is an endemic and native plant from Brazil. It is found mainly in the Atlantic Forest region, in Paraná and São Paulo states. This study aimed to evaluate the phytochemical composition using a qualitative phytochemical analysis and high performance liquid chromatography with diode array detector (HPLC-DAD), to evaluate preliminary toxicity in vitro using Artemia salina and antioxidant potential. Qualitative phytochemical analysis revealed the presence of alkaloids, flavonoids, iridoids, steroids, triterpenes, saponinic glycosides and aminogroups. The analysis by HPLCDAD provided the characteristic fingerprint of each sample. In the $A$. salina assay, the results of $L_{50}$ over $1000 \mu \mathrm{g} / \mathrm{mL}$ for all samples did not indicate toxicity of the evaluated extracts. All samples demonstrated antioxidant activity by reducing phosphomolybdenum complex. The highest activity was detected in the chloroform fraction, which presented activity of $92.51 \%$ compared to the standard rutin. Regarding the trial to reduce the radical $\mathrm{DPPH} \cdot$, the ethyl acetate fraction showed an $\mathrm{IC}_{50}$ of $26.98 \mu \mathrm{g} / \mathrm{mL}$. In the thiobarbituric acid assay (TBARS), the hexane fraction showed the highest antioxidant activity compared to the standard rutin. These findings indicate that the Senecio westermanii is a potential source of natural antioxidants, stimulating
\end{abstract}

Recebido para publicação em 16/10/2014

Aceito para publicação em 15/04/2015

10.1590/1983-084X/14_137

Rev. Bras. PI. Med., Campinas, v.17, n.4, supl. III, p.1031-1040, 2015. 
new studies that enable their use in the treatment of disorders associated with free radicals. In addition, the species did not present toxicity in its evaluation, ensuring its applicability.

Keywords: Senecio; Phytochemical analysis; Artemia salina; Antioxidant; HPLC-DAD

\section{INTRODUÇÃO}

Estudos realizados com espécies do gênero Senecio L. evidenciaram a presença de flavonoides com a capacidade de reduzir a ação oxidante de substâncias reativas nas espécies Senecio gallicus, Senecio candicans e Senecio erraticus (Mansour \& Saleh, 1981; Mezache et al., 2009; Hariprasath et al., 2012). Além dos flavonoides, Silva et al. (2006) evidenciaram a presença de alcaloides pirrolizidínicos, sendo esta classe de metabólito secundário considerado um marcador quimiossistemático para a tribo Senecioneae.

Segundo Lima \& Bezerra (2012) os flavonoides são metabólitos que atuam na proteção do sistema biológico contra o efeito nocivo de processos ou reações que podem causar oxidação no organismo humano, prevenindo ou amenizando doenças. Os alcaloides pirrolizidínicos são conhecidos pela sua atividade hepatotóxica caracterizada por hepatomegalocitose, fibrose e proliferação de ductos biliares (Barros et al., 2007).

Frescura et al. (2013) destacam a importância dos antioxidantes naturais, a exemplo dos flavonoides, em função das suas diversas atividades farmacológicas e terapêuticas. A ação desses metabólitos está relacionada com a melhora da perda da acuidade visual e alterações do campo de visão. Sua presença ameniza os sintomas de insuficiência dos vasos linfáticos e venosos associados com algumas doenças hemorrágicas. Além disso, promovem a normalização da permeabilidade e resistência da parede dos vasos no controle da hipertensão arterial (Frescura et al., 2013).

Os polifenóis em especial os flavonoides possuem ação terapêutica principalmente ligada a patologias que se desenvolvem através dos radicais livres, porém outras atividades como antihiperlepidêmica, anticarcinogênica e antiinflamatória foram encontradas (Becho et al., 2009). A principal propriedade dos antioxidantes é sua capacidade de neutralizar ou sequestrar os radicais livres, tanto na etapa inicial como na propagação do processo oxidativo (Sousa et al., 2007).

Alguns antioxidantes apresentam a capacidade de eliminar espécies de radicais livres, tais como o superóxido e hidroxil, além de reduzir radicais tocoferóis de volta para sua forma ativa nas membranas celulares, mantendo a sua integridade em células dos organismos aeróbios (Oliveira et al., 2011). Também podem prevenir mutações em DNA de humanos, uma vez que altas concentrações dela reduzem mutações causadas por estresse oxidativo em células humanas in vitro (Lutsenko et al., 2002). Sua presença nos alimentos tem um papel importante na preservação de nutrientes do alimento bem como no aumento do tempo de vida em prateleira. Além disso, é um nutriente essencial que deve ser ingerido diariamente (Chaves et al., 2004).

Porém muitos dos antioxidantes utilizados pelas indústrias alimentícias são sintéticos, como, por exemplo, o BHT. A estrutura fenólica do BHT possui a capacidade de doar um elétron a um radical livre, evitando dessa forma a ação destes radicais sobre os alimentos (Ramalho \& Jorge, 2006).

Ensaios de toxicidade são frequentemente empregados para averiguar a segurança do uso de plantas na medicina popular, assim como extratos e outros insumos obtidos através de produtos naturais. Para Meyer et al. (1982) a utilização do microcrustáceo Artemia salina é frequentemente aplicado em ensaios de toxicidade aguda para pesquisa preliminar de atividade biológica de extratos e frações obtidos a partir de produtos naturais. Esse simples organismo pode ser usado como um monitor conveniente para a citotoxicidade de produtos, além de ser um método rápido, simples e sensível a substâncias tóxicas.

Devido a ausência de estudos referentes a Senecio westermanii Dusén e por não ser utilizada pela população como fitoterápico ou alimento, a espécie despertou um grande interesse em ser estudada devido ao gênero possuir metabólitos secundários de interesse biológico, como alcaloides e flavonoides. Dessa forma, o objetivo deste trabalho foi realizar a caracterização fitoquímica do extrato bruto etanólico e frações das partes aéreas (folha e caule) da planta, com relação ao potencial antioxidante in vitro e avaliar a toxicidade das amostras frente a Artemia salina.

\section{MATERIAL E MÉTODOS Material Vegetal}

As partes aéreas da espécie Senecio westermanii foi coletada na Estrada da Graciosa, localizada no município de Quatro Barras - PR, nas coordenadas $25^{\circ} 18^{\prime} 27^{\prime \prime} \mathrm{S}$ e $48^{\circ} 56^{\prime} 37^{\prime \prime} \mathrm{W}, 845 \mathrm{~m}$. A identificação da espécie vegetal foi realizada no

Rev. Bras. PI. Med., Campinas, v.17, n.4, supl. III, p.1031-1040, 2015. 
Museu Botânico Municipal (MBM) de Curitiba-PR, onde está depositada a exsicata MBM 379066. O projeto foi regularizado de acordo com a resolução n³5, de 27 de abril de 2011 do Ministério do Meio Ambiente que dispõe sobre a regularização de atividades de acesso ao patrimônio genético.

\section{Obtenção do extrato bruto e frações}

O material coletado foi seco em temperatura ambiente e triturado em moinho de facas e martelo. A partir de $6,3 \mathrm{~kg}$ do material vegetal seco e estabilizado, o extrato bruto etanólico $(355,98 \mathrm{~g})$ foi obtido por meio de extração etanólica exaustiva em aparelho de Soxhlet e posteriormente concentrado em evaporador rotatório, com pressão reduzida, a temperatura de $65{ }^{\circ} \mathrm{C}$ e $150 \mathrm{rpm}$. A partir do extrato bruto foram obtidas as frações n-hexano $(57,98 \mathrm{~g})$, clorofórmio $(80,04 \mathrm{~g})$, acetato de etila $(3,36 \mathrm{~g})$ e hidroalcoólica remanescente $(291,73$ g) por partição líquido/líquido com solventes de polaridade crescente (Carvalho et al., 2009). Para o extrato aquoso foram macerados à quente $40 \mathrm{~g}$ do material vegetal em $200 \mathrm{~mL}$ de água destilada a $70^{\circ} \mathrm{C}$ em banho-maria por um período de uma hora (Moreira, 1979).

\section{Estudo fitoquímico}

As frações do extrato hidroalcoólico da espécie S. westermanii foram submetidas ao ensaio fitoquímico de acordo com Moreira (1979). Os grupos químicos pesquisados foram os alcaloides; flavonoides; cumarinas; iridoides; antraquinonas; esteroides e/ou triterpenos. Para o extrato aquoso foram avaliadas a presença de heterosídeos antociânicos, saponínicos, cianogênicos; taninos hidrolisáveis e condensados; ácidos fixos e voláteis; e aminogrupos.

\section{Análises por CLAE-DAD}

As análises cromatográficas foram realizadas empregando cromatógrafo líquido de alta eficiência modelo: Merck Hitachi, detector de arranjo de diodos (DAD) L-2450, com varredura entre 220-400 nm. Coluna X-Terra RP-18 $(25 \mathrm{~cm} x$ $4,6 \mathrm{~mm} \times 5 \mu \mathrm{m})$ e pré-coluna $(2,5 \mathrm{~cm} \times 3 \mathrm{~mm}) \mathrm{de}$ mesma fase da coluna. A eluição foi realizada com gradiente de concentração composta por fase $A$ (fase ácida) e fase $\mathrm{B}$ (metanol) iniciando com $80 \%$ de fase $A$ e $20 \%$ da fase $B$ ( 0 a 35 min.) até $100 \%$ de fase $B$ isocrático até 45 min com a vazão de fluxo da bomba de $1 \mathrm{~mL}$. $\mathrm{min}^{-1}$ e volume injetado de $20 \mu \mathrm{L}$.

\section{Tocixidade frente ao microcrustáceo Artemia salina}

Foram adquiridos os ovos do microcrustáceo da espécie Artemia salina e procedeu-se ao ensaio conforme metodologia desenvolvida por Meyer et al.
(1982). Os ovos do crustáceo foram eclodidos em água salina, preparada com $14,31 \mathrm{~g}$ de sal marinho e disolvido em $400 \mathrm{~mL}$ de água purificada. Para esta quantidade, foram adicionados $200 \mathrm{mg}$ dos ovos do crustáceo. O pH foi mantido entre 8,0 e 9,0 para evitar a morte dos crustáceos. A temperatura foi controlada entre $27^{\circ} \mathrm{C}$ e $30^{\circ} \mathrm{C}$ e a solução foi mantida sob agitação e aeração constante por 48 horas. Nas primeiras horas do processo foi mantida iluminação $(20 \mathrm{~W})$ sobre o recipiente. Foram preparadas as amostras em metanol nas concentrações de $1000 \mu \mathrm{L} / \mathrm{mL}, 100 \mu \mathrm{L} / \mathrm{mL}$ e 10 $\mu \mathrm{L} / \mathrm{mL}$ e adiconados 10 microcrustáceos. Como controle positivo foi utilizado sulfato de quinidina para ocasionar a morte da A. salina. Após 24 horas de contato dos nauplios realizou a contagem dos crustáceos mortos. Os dados foram submetidos a análise pelo método estatístico Probitos e determinados os valores da dose letal $\left(\mathrm{DL}_{50}\right) \mathrm{com}$ $95 \%$ de intervalo de confiança.

\section{Avaliação da atividade antioxidante}

\section{Ensaio de redução do complexo fosfomolibdênio}

A reação para formação do complexo requer o preparo de um reativo que consiste de uma solução com fosfato de sódio $0,1 \mathrm{~mol} / \mathrm{L}(28 \mathrm{~mL})$, molibdato de amônio $0,03 \mathrm{~mol} / \mathrm{L}(12 \mathrm{~mL}$ ) e ácido sulfúrico $3 \mathrm{~mol} / \mathrm{L}$ $(20 \mathrm{~mL})$, sendo o volume final ajustado $\mathrm{H}_{2} \mathrm{O}$ para 100 $\mathrm{mL}$. O extrato bruto e as frações foram preparados na concentração de $200 \mu \mathrm{g} / \mathrm{mL}$ em metanol, assim como os padrões utilizados, vitamina $\mathrm{C}$ e rutina. Em tubos de ensaio foram colocados alíquotas de 0,3 $\mathrm{mL}$ de cada amostra e adicionado $3 \mathrm{~mL}$ de solução reagente do complexo fosfomolibdênio. Os tubos foram fechados e incubados a $95^{\circ} \mathrm{C}$ por 90 minutos. Após resfriamento, a leitura das absorbâncias foi realizada em espectrofotômetro UV-1601 PC Shimadzu ${ }^{\circledR}$ no comprimento de onda $695 \mathrm{~nm}$. O branco era constituído de $0,3 \mathrm{~mL}$ de metanol e $3 \mathrm{~mL}$ de reativo. Todo o ensaio foi realizado em triplicata (Prieto et al., 1999).

A capacidade antioxidante das amostras foi expressa em atividade antioxidante relativa (AAR) em relação aos padrões, conforme a equação:

\section{AAR(\%): (Abs amostra - Abs branco $) \times 100$}

(Abs padrão - Abs branco)

A partir dos resultados de AAR(\%) realizouse a análise estatística dos resultados pelo teste de Scott-Knott $(1974)(p<0,05)$.

\section{Redução do radical DPPH•}

A avaliação do potencial de redução do radical DPPH• foi realizada de acordo com a

Rev. Bras. PI. Med., Campinas, v.17, n.4, supl. III, p.1031-1040, 2015. 
metodologia proposta por Mensor et al. (2001). Para cada amostra do extrato bruto, frações e dos padrões vitamina $C$ e rutina, foram preparadas concentrações crescentes as quais ficaram em meio reacional com $1 \mathrm{~mL}$ de solução de DPPH a $0,03 \mathrm{mmol} / \mathrm{mL}$. Para cada amostra foi preparado um branco com 2,5 mL da solução da amostra e $1 \mathrm{~mL}$ de metanol para cada concentração. Paralelamente foi realizado um controle com $2,5 \mathrm{~mL}$ de metanol e $1 \mathrm{~mL}$ de DPPH. Após 30 minutos as absorbâncias foram medidas em espectrofotômetro a $518 \mathrm{~nm}$.

A atividade sequestrante do radical $\mathrm{DPPH}$ foi expressa em porcentagem de atividade antioxidante (AA), conforme a equação:

\section{$\mathrm{AA}(\%): 100$ - (Abs amostra - Abs branco $)$ \\ (Abs controle)}

Para comparar os resultados obtidos foi estabelecida a concentração inibitória $\left(\mathrm{IC}_{50}\right)$, calculada por regressão linear. No intervalo linear foi estabelecida a equação da reta do tipo $y=a x+b$, sendo dessa forma, determinada para cada amostra analisada os valores de $\mathrm{IC}_{50}$. Para a análise estatística dos resultados foi utilizado o teste de Scott-Knott $(1974)(p<0,05)$.

\section{(TBARS)}

Substâncias reativas ao ácido tiobarbitúrico

O ensaio foi realizados de acordo com Morais et al. (2006). Em tubos de ensaio, foram adicionados $0,5 \mathrm{~mL}$ de solução de gema de ovo $(10 \% \mathrm{p} / \mathrm{v}), 0,1 \mathrm{~mL}$ de amostra ou padrão (BHT) $1000 \mathrm{ppm}$ e o volume foi completado com água destilada para $1 \mathrm{~mL}$. Em seguida, foi acrescentado a cada um dos tubos de ensaio $0,05 \mathrm{~mL}$ de solução de 2,2'-azobis(2-amidinopropano) dihidrocloreto (ABAP) 0,07 mol.L-1, $1,5 \mathrm{~mL}$ de solução de ácido acético $20 \%$ (pH 3,5) e 1,5 mL de solução de ácido tiobarbitúrico (TBA) 0,8\% p/v em solução de dodecil sulfato de sódio (SDS) 1,1\% p/v. Os tubos de ensaio foram submetidos ao banho-maria $\left(95^{\circ} \mathrm{C}\right)$ por 1 hora. Após resfriamento, foi acrescentado $5 \mathrm{~mL}$ de n-butanol a cada tubo, que foi centrifugado durante 10 minutos a $3000 \mathrm{rpm}$. Os sobrenadantes foram submetidos ao espectrofotômetro em 532 nm.

A atividade antioxidante foi determinada pelo Índice Antioxidante (IA), segundo equação:

\section{IA(\%): (1 - Abs amostra $) \times 100$ (Abs controle)}

A partir dos reultados de $\mathrm{IA}(\%)$ realizou-se a análise estatística dos resultados pelo teste de Scott-Knott $(1974)(p<0,05)$.

\section{RESULTADOS E DISCUSSÃO}

O resultado do estudo fitoquímico qualitativo indicou por meio de reações características para o extrato hidroalcoólico a presença de alcaloides (Mayer, Dragendorff e Bertrand), flavonoides (leucoantocianidinas, heterosídeos flavônicos e oxálico bórico), iridoides (floroglucinol e ácido sulfúrico/vanilina) e esteroides/triterpenos (Liberman-Bouchard e Keller-Kelliani) (Tabela 1). Porém para o extrato aquoso constatou-se heterosídeos saponínicos (teste de formação de espuma) e aminogrupos (Tabela 2). Através desses ensaios a espécie em estudo revelou um perfil químico semelhante ao encontrado para outras espécies do gênero.

O perfil cromatográfico do extrato bruto etanólico e frações das partes aéreas de $S$. westermanii foram comparados entre si empregando como parâmetros o tempo de retenção (Tabela 3) e o espectro de absorção molecular obtido através do detector de arranjo de diodos. Os sinais que apresentaram similaridade com relação aos parâmetros monitorados para as amostras foram numerados. Os picos foram tratados como sinais, pois pode haver mais de uma substância em um mesmo sinal.

Analisando o cromatograma das amostras

TABELA 1. Resultado da análise fitoquímica a partir do extrato hidroalcoólico da espécie $S$. westermanii

\begin{tabular}{lcccc}
\hline Grupos Fitoquímicos & FH & FCL & FAE & FHR \\
\hline Alcaloides & - & + & - & - \\
Flavonoides & - & + & + & + \\
Cumarinas & - & - & - & - \\
Iridoides & + & - & - & - \\
Antraquinonas & - & - & - & - \\
Esteroides/Triterpenos & + & - & - & - \\
\hline
\end{tabular}

Fração Hexano (FH), Fração Clorofórmio (FCL), Fração Acetato de Etila (FAE), Fração Hidroalcoólica Remanescente (FHR); (+) positivo, (-) negativo

Rev. Bras. PI. Med., Campinas, v.17, n.4, supl. III, p.1031-1040, 2015. 
TABELA 2. Resultado da análise fitoquímica do extrato aquoso da espécie $S$. westermanii

\begin{tabular}{lc}
\hline Grupos Fitoquímicos & Extrato Aquoso \\
\hline Heterosídeos antociânicos & - \\
Heterosídeos saponínicos & + \\
Heterosídeos cianogênicos & - \\
Taninos hidrolisáveis & - \\
Taninos condensados & - \\
Ácidos fixos & - \\
Ácidos voláteis & - \\
Aminogrupos & + \\
\hline
\end{tabular}

(+) positivo, (-) negativo

(Figuras 1-4) observa-se que o sinal 1 está presente no extrato bruto etanólico e na fração acetato de etila. O sinal 2 está presente no extrato bruto etanólico e fração hidroalcoólica remanescente. $O$ sinal 3 presente apenas no extrato bruto etanólico. O sinal 4 está presente no extrato bruto etanólico e na frações clorofórmio e acetato de etila. O sinal 5 está presente apenas na fração clorofórmio. O sinal 6 está presente apenas na fração clorofórmio. E os sinais 7, 8 e 9 estão presentes, respectivamente, nas frações clorofórmio, acetato de etila e hidroalcoólica remanescente.

Apesar dos tempos de retenção semelhantes para os demais sinais presentes nos cromatogramas, porém não apresentaram similaridade quanto ao espectro de absorção molecular indicando que são substâncias diferentes.

De acordo com Araújo et al. (2010) o bioensaio de toxicidade frente a Artemia salina possui o intuito de monitorar extratos vegetais em diversos laboratórios de produtos naturais. Porém diversos ensaios biológicos simples têm sido desenvolvidos para a mesma função, mas a $A$. salina tem sido inclusa na rotina laboratorial para monitorar o estudo de extratos e frações de plantas, além disso para auxiliar no isolamento, purificação e elucidação estrutural de componentes isolados dessas amostras. $\mathrm{O}$ ensaio permite a avaliação da toxicidade geral e é considerado essencial como bioensaio preliminar no estudo de amostras com potencial atividade biológica.

Bednarczuk et al. (2010) comentam que a $A$. salina são bastante utilizadas em ensaios toxicológicos por serem de fácil manuseio, baixo custo, fácil cultivo e obtenção. Os ensaios de letalidade são largamente empregados em testes toxicológicos podendo-se obter a dose letal mediana $\left(\mathrm{DL}_{50}\right)$, dose necessária para ocasionar a morte de $50 \%$ dos microcrustáceos nas amostras em estudo.

Dessa forma para o extrato bruto etanólico e frações das partes aéreas de $S$. westermanii no ensaio de toxicidade ao microcrustáceo $A$. salina obteve-se uma $\mathrm{DL}_{50}$ superior a $1000 \mu \mathrm{g} / \mathrm{mL}$ para todas as amostras analisadas. De acordo com Nguta et al. (2011) amostras com valores de $\mathrm{DL}_{50}$ inferiores $100 \mu \mathrm{g} / \mathrm{mL}$ são considerados altamente tóxicas, valores entre 100 e $500 \mu \mathrm{g} / \mathrm{mL}$ são moderadamente tóxicas, entre 500 e $1000 \mu \mathrm{g} / \mathrm{mL}$ são suavemente tóxicas e acima de $1000 \mu \mathrm{g} / \mathrm{mL}$ são atóxicas.

Estudos realizadas com algumas espécies do gênero Senecio mostraram se tóxicas frente ao microcrustáceo como exposto por Bardón et al. (2007), onde os extratos clorofórmico e metanólico da mistura das folhas e flores da espécie Senecio santelisis apresentaram uma $\mathrm{DL}_{50}$ de 49 e $1 \mu \mathrm{g} / \mathrm{mL}$, respectivamente. E para a espécie Senecio puchii os extratos clorofórmico e metanólico apresentaram uma $\mathrm{DL}_{50}$ de 109 e $202 \mu \mathrm{g} / \mathrm{mL}$, respectivamente (Bardón et al., 2007). Apesar dos alcaloides

TABELA 3. Tempo de retenção dos sinais monitorados presentes nas análises das frações e extrato bruto hidroalcóolico de S. westermani

\begin{tabular}{llll}
\hline Pico & Amostras & $\lambda_{\text {Abs }}$ & TR \\
\hline 1 & EB, FAE & $281 ; 328$ & 19,74 \\
2 & EB, FHR & $284 ; 326$ & 20,38 \\
3 & EB & $286 ; 326$ & 21,27 \\
4 & EB, FCL, FAE & $284 ; 328$ & 21,65 \\
5 & FCL & 310 & 13,62 \\
6 & FCL & $280 ; 308$ & 25,62 \\
7 & FCL & $280 ; 315$ & 26,38 \\
8 & FAE & $256 ; 294 ; 350$ & 20,29 \\
9 & FHR & $292 ; 326$ & 14,39 \\
\hline
\end{tabular}

$\lambda_{\text {Abs }}$ : Bandas de absorção (nm); TR: Tempo de Retenção (min) 


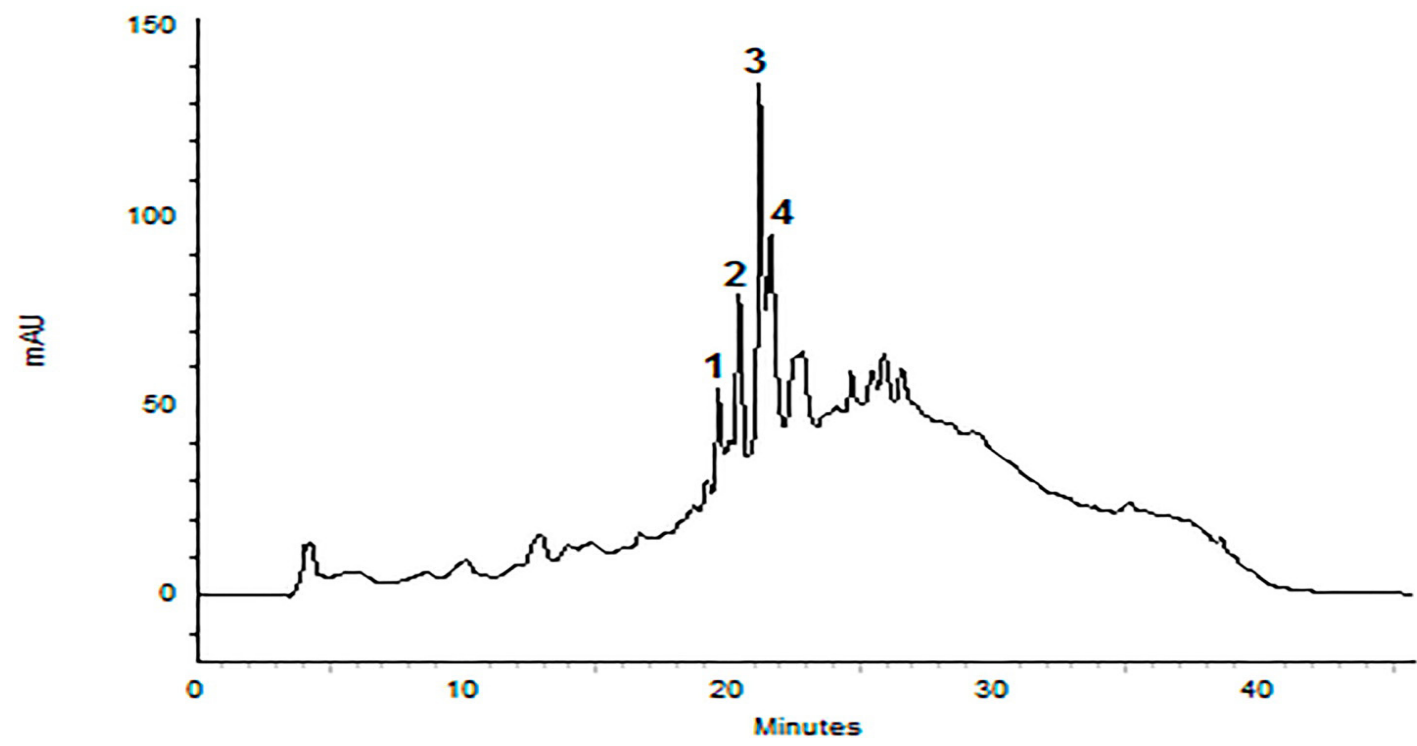

FIGURA 1. Perfil cromatográfico do extrato bruto etanólico das partes aéreas de $S$ westermanii

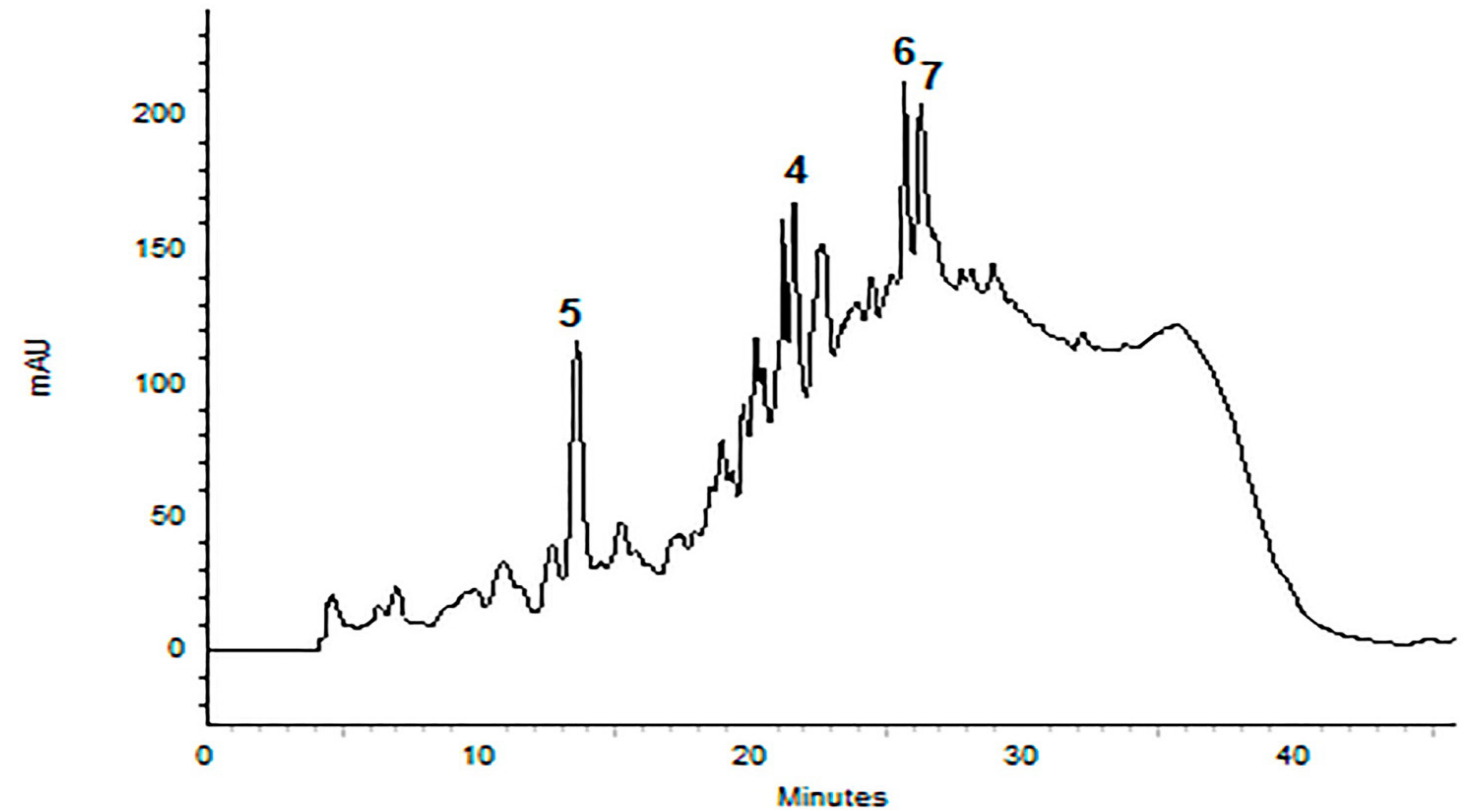

FIGURA 2. Perfil cromatográfico da fração clorofórmio das partes aéreas de $S$ westermanii

pirrolizidínicos extraídos das espécies do gênero Senecio demonstrarem toxicidade em bovinos (Barros et al., 2007; Lucena et al., 2010), mas de acordo com Bolzan et al. (2007) esses metabólitos não estão presentes em todas as espécies do gênero.

Dessa forma, algumas espécies do gênero não apresentaram atividade tóxica preliminar frente ao microcrustáceo $A$. salina como expôs Bardón et al. (2007) para os extratos clorofórmico e metanólico das folhas de Senecio leucostachys e Cuadra et al. (2005) para o extrato etanólico das folhas de Senecio miser. Com isso, o extrato bruto etanólico e as frações da espécie em estudo não apresentaram toxicidade preliminar para a $A$. salina apresentando uma $\mathrm{DL}_{50}>1000 \mu \mathrm{g} / \mathrm{mL}$, quando comparada ao sulfato de quinidina com valor de $\mathrm{DL}_{50}$ em $89,41 \mu \mathrm{g} /$ $\mathrm{mL}$.

Para Campos \& Frasson (2011) definir uma metodologia mais eficiente de extração de compostos antioxidantes pode não ser fácil, pois estes compostos podem sofrer influência de diversos fatores como a natureza do vegetal, tamanho das partículas, solvente utilizado, tempo e temperatura de extração. Com isso, há diversos métodos para a extração dos componentes antioxidantes em vegetais, dentre eles os tradicionais métodos com solventes orgânicos como a água, etanol, éter e metanol. Para avaliar o desempenho da atividade antioxidante do extrato bruto e frações da espécie S. westermanii foram utilizados os métodos fosfomolibdênio, DPPH e TBARS. 


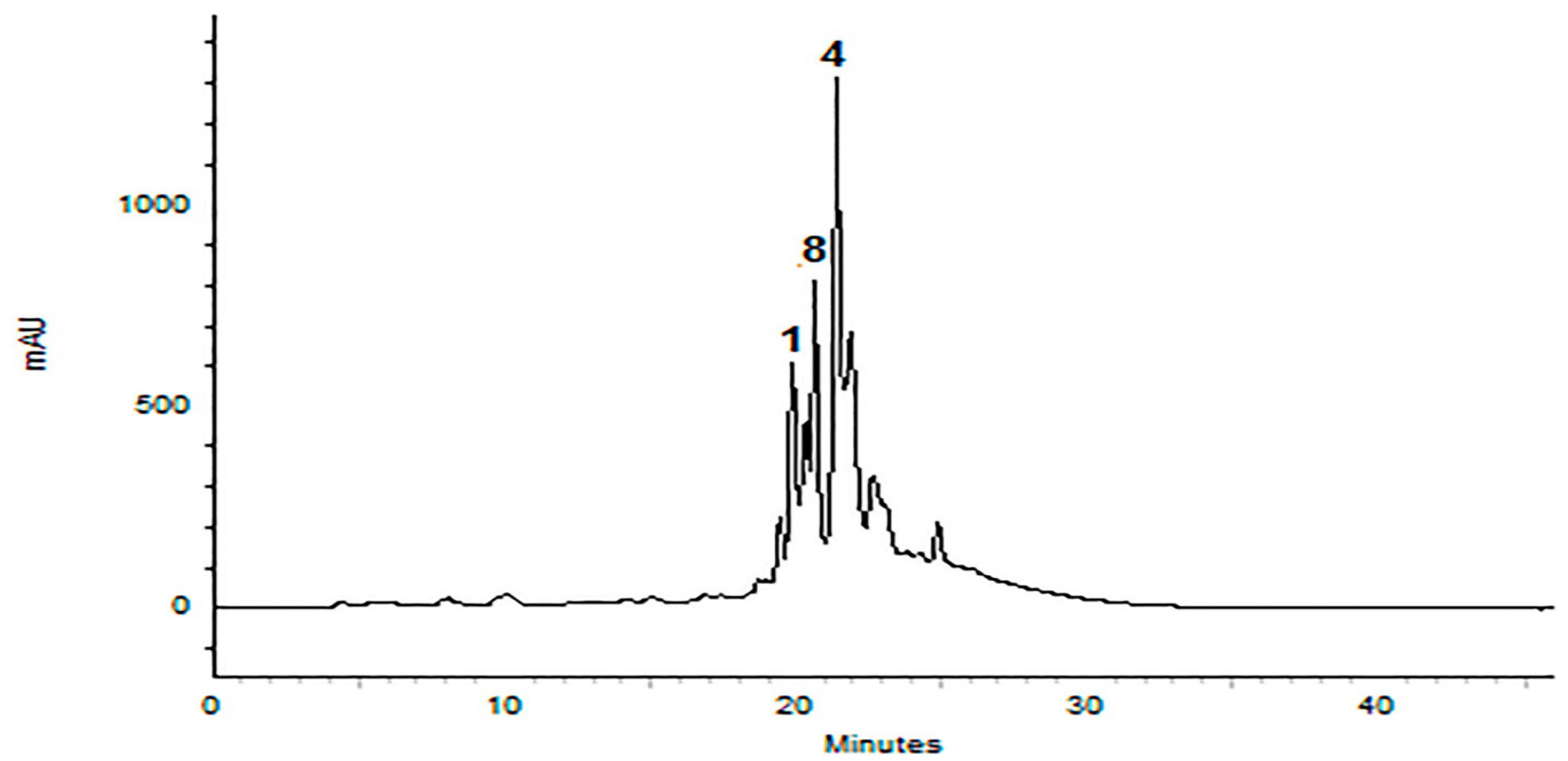

FIGURA 3. Perfil cromatográfico da fração acetato de etila das partes aéreas de $S$ westermanii

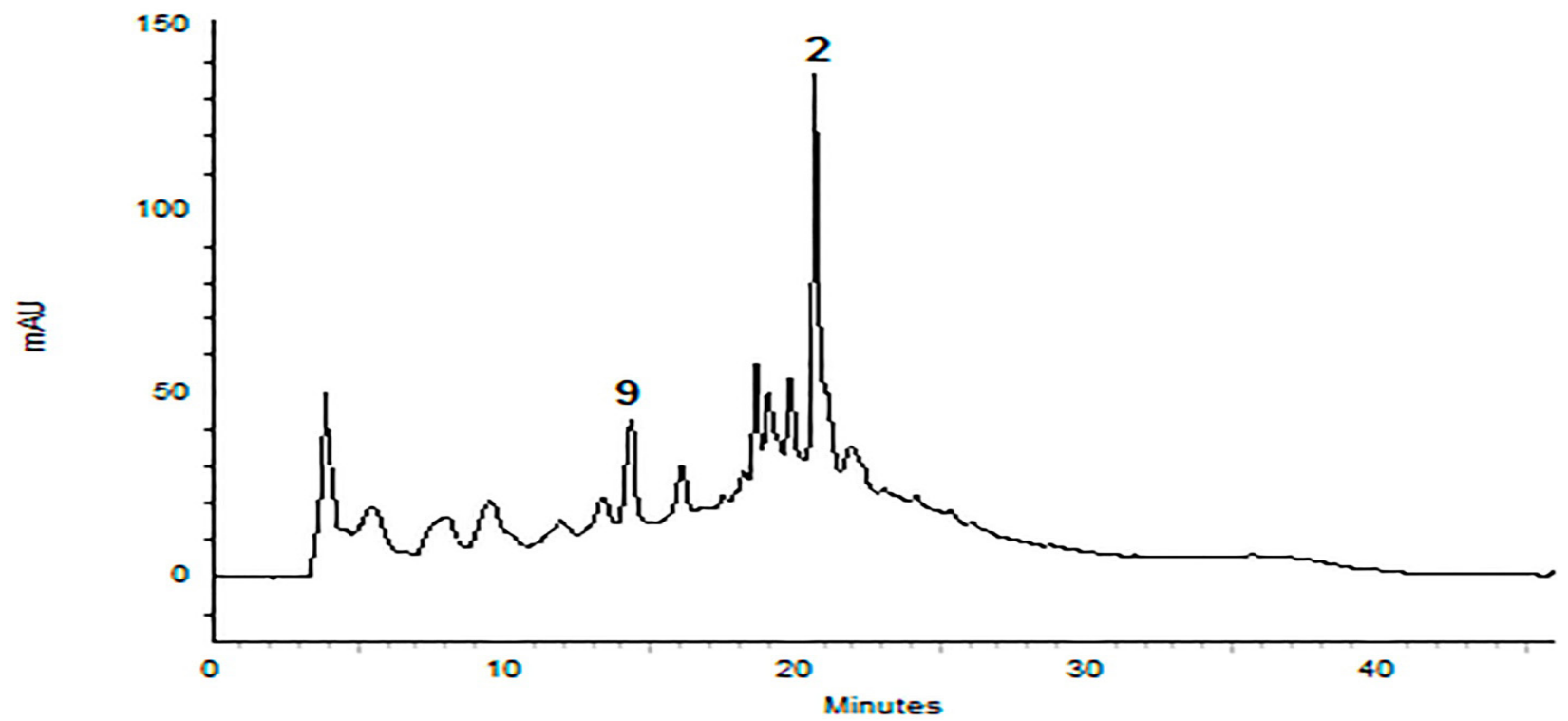

FIGURA 4. Perfil cromatográfico da fração hidroalcoólica das partes aéreas de $S$ westermanii

Para Martelli \& Nunes (2014) os antioxidantes são substâncias que retardam a velocidade da oxidação, inibindo os radicais livres e prevenindo a formação de doenças, tornando-se essenciais para o equilíbrio entre os radicais livres e o sistema de defesa antioxidante do corpo humano. De acordo com Sousa et al. (2007) os radicais livres e outros oxidantes são considerados como grandes causadores de doenças para o ser humano ocasionando declínio do sistema imune, câncer, doenças cardiovasculares, catarata, disfunções cerebrais e diabetes mellitus tipo I. Dessa forma, uma dieta baseada em substâncias essenciais tem um papel importante na prevenção dessas doenças devido a combinação de substâncias presentes nos vegetais, reduzindo o risco de desenvolvimento de doenças crônicas (Pereira \& Cardoso, 2012).

De acordo com Prieto et al. (1999) a avaliação pelo método fosfomolibdênio possui a vantagem de avaliar a capacidade antioxidante de componentes lipofílicos e hidrofílicos. Além disso, o método utilizado permite avaliar a capacidade total de uma mistura complexa de compostos (extratos e frações obtidos de plantas) de maneira simples e barata. A partir dos resultados obtidos através desse método (Tabela 4) observa-se que a fração clorofórmio demonstrou potencial antioxidante, com atividade de 92,51\% em comparação com a atividade 
da rutina, utilizada como controle positivo. Segundo Balestrin et al. (2008) a atividade antioxidante no ensaio de fosfomolibdênio pode estar relacionada a uma mistura complexa de componentes químicos, ocorrendo um sinergismo entre eles.

As substâncias existentes no extrato bruto etanólico e frações da espécie $S$. westermanii também possuem a capacidade em reagir com o radical livre instável DPPH • e convertê-lo no 2,2-difenil-1-picril hidrazina. A capacidade ou a atividade de atuar como um antioxidante é visível quando há mudança de cor nas soluções preparadas. A fração acetato de etila foi a amostra que mais se aproximou da atividade antioxidante comparada aos controles vitamina $\mathrm{C}$ e rutina (Tabela 4), assim como as outras frações e o extrato bruto. A atividade antioxidante medida através do radical DPPH • mostra a capacidade de substâncias pertencentes ao extrato e frações das partes aéreas de $S$. westermanii em sequestrar radicais livres do meio existente.

De acordo com Andrade et al. (2007) substâncias com núcleo fenólico, como flavonoides, ácidos fenólicos e o tocoferol atuam como captadores de espécies reativas de oxigênio, desta forma possuem características antioxidantes. Os flavonoides atuam como antioxidantes primários, sendo as flavonas e os flavonóis as classes mais encontradas nas plantas. Porém, dependendo do estado de oxidação da cadeia heterocíclica dos flavonoides, tem-se inúmeras atividades biológicas relacionadas a esse metabólito secundário, como atividade antiinflamatória, antitumoral, agregação plaquetária, redução da fragilidade e permeabilidade capilar, inibição na destruição do colágeno e redução na incidência de doenças cardiovasculares (Pereira \& Cardoso, 2012). Além disso os flavonoides são comunente isolados da fração acetato de etila como exposto por Paula et al. (2014) e Hirota et al. (2012). Isso explica a uma maior atividade antioxidante da fração acetato de etila da espécie $S$. westermanii.

No ensaio de peroxidação lipídica pelo método TBARS observa-se que as frações hexano e hidroalcoólica remanescente tiveram atividade antioxidante semelhante ao controle BHT (Tabela 4). Observando-se que a fração hexânica possui um melhor resultado devido sua característica lipofílica, permitindo uma melhor interação com a matriz lipídica. Porém, Santi et al. (2014) comentam que os compostos fenólicos em vegetais abrange uma gama de substâncias, desde moléculas simples até aquelas com alto grau de polimerização, comprovando a atividade antioxidante dos ácidos fenólicos na inibição da peroxidação lipídica.

A peroxidação lipídica promove a perda da fluidez da membrana plasmática e a liberação de proteínas intracelulares, ocasionando lesões de tecidos e afetando biomoléculas no organismo humano (Júnior \& Pereira, 2008). Além disso pode ocorrer inativação enzimática, ruptura de membrana, aumento da aterogenicidade de lipoproteínas plasmáticas, mutações e à morte celular (Cerqueira et al., 2007).

Apesar da grande produção de antioxidantes em indivíduos saudáveis, os componentes celulares não são protegidos por antioxidantes endógenos em sua totalidade, sendo necessário a busca de antioxidantes exógenos para a complementação do sistema de defesa, isso faz com que o organismo seja protegido de substâncias oxidantes (Cerqueira et al., 2007).

A inibição da peroxidação lipídica é uma das principais preocupações sobre a questão do estresse oxidativo, a inibição está relacionada com a ação dos fitosteróis no corpo humano, onde agem como transportadores de elétrons. Por serem estruturalmente semelhantes aos lípides de membrana, os fitoesteróis agem na cadeia de transporte de elétrons evitando a oxidação celular do organismo, agindo quimicamente como antioxidantes em membranas celulares e estabilizando-as (Yoshida \& Nikki, 2003).

Os fitoesteróis são constituintes das membranas celulares das plantas e são biologicamente comparáveis ao colesterol nos animais, apenas diferem por conterem grupos metilo ou etilo na cadeia lateral da molécula (Ellegard et al., 2007).

Através da análise fitoquímica preliminar foi possível identificar classes de metabólitos secundários de interesse farmacológico presentes nos extrato bruto etanólico e frações de $S$. westermanii. Para complementar foram realizados fingerprint das amostras através da análise por CLAE-DAD para comprovar a presença de vários componentes secundários nas amostras analisadas, observando-se que alguns sinais possuem o mesmo tempo de retenção e espectro de absorção para mais de uma amostra.

Para auxiliar de forma complementar os estudos fitoquímicos, utilizou-se o bioensaio de toxicidade sobre $A$. salina, isentando o extrato bruto etanólico e as frações de apresentarem toxicidade preliminar. Além disso as amostras analisadas de $S$. westermanii mostrou-se excelente perante a atividade antioxidante por diferentes mescanismos, como capacidade redutora (complexo fosfomolibdênio), capacidade de sequestro de radicais livres (DPPH) e efeito protetor contra a peroxidação lipídica (TBARS). Aliado a isso, temse a ausência de toxicidade frente à $A$. salina mostrando que a espécie em estudo possui potencial para o desenvolvimento de fitoterápicos, uma vez que outras espécies do mesmo gênero, cuja

Rev. Bras. PI. Med., Campinas, v.17, n.4, supl. III, p.1031-1040, 2015. 
TABELA 4. Análise antioxidante utilizando os métodos Fosfomolibdênio, DPPH e TBARS do extrato bruto e frações das partes aéreas de S. westermani

\begin{tabular}{|c|c|c|c|c|}
\hline \multirow[b]{2}{*}{ Amostra } & \multicolumn{2}{|c|}{ REDUÇÃO DO COMPLEXO FOSFOMOLIBDÊNIO } & \multirow{2}{*}{$\begin{array}{c}\text { REDUÇÃO DO } \\
\text { RADICAL DPPH } \\
I_{50}(\mu \mathrm{g}) \pm \mathrm{DP}\end{array}$} & \multirow{2}{*}{$\begin{array}{c}\text { TBARS } \\
\mathrm{A}(\%) \pm \mathrm{DP}\end{array}$} \\
\hline & $\begin{array}{c}\text { Atividade Antioxidante em } \\
\text { Relação à Rutina - AA (\%) } \\
\pm \text { DP }\end{array}$ & $\begin{array}{c}\text { Atividade Antioxidante } \\
\text { em Relação à Vitamina } \\
\text { C - AA (\%) } \pm \text { DP }\end{array}$ & & \\
\hline BHT & - & - & - & $33,98 \pm 6,11 n$ \\
\hline Vitamina C & - & $100 \mathrm{~d}$ & $4,92 \pm 0,07 \mathrm{~h}$ & - \\
\hline Rutina & $100 \mathrm{a}$ & - & $6,15 \pm 0,08 \mathrm{~h}$ & - \\
\hline EB & $24,33 \pm 0,02 c$ & $8,70 \pm 0,02 \mathrm{~g}$ & $169,52 \pm 3,50$ & $3,68 \pm 7,80 p$ \\
\hline $\mathrm{FH}$ & $64,44 \pm 0,04 b$ & $23,04 \pm 0,04 \mathrm{f}$ & $346,96 \pm 1,83 \mathrm{~m}$ & $23,04 \pm 10,820$ \\
\hline FCL & $92,51 \pm 0,04 a$ & $33,08 \pm 0,04 \mathrm{e}$ & $71,46 \pm 0,21 j$ & $1,05 \pm 8,51 p$ \\
\hline FAE & $53,74 \pm 0,02 b$ & $19,22 \pm 0,02 f$ & $26,98 \pm 0,77 i$ & $4,85 \pm 10,35 p$ \\
\hline FHR & $20,05 \pm 0,02 c$ & $7,17 \pm 0,02 \mathrm{~g}$ & $146.72 \pm 0,12 \mathrm{k}$ & $15,50 \pm 6,44 \circ$ \\
\hline
\end{tabular}

Extrato Bruto (EB), Fração Hexano (FH), Fração Clorofórmio (FCL), Fração Acetato de Etila (FAE), Fração Hidroalcoólica Remanescente

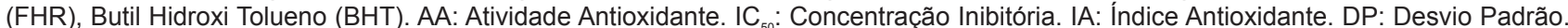
${ }^{*}$ Resultados seguidos pela mesma letra não diferem estatisticamente $(p<0,05)$ entre si, pelo teste de Scott-Knott $(1974)$.

rota metabólica é semelhante, tem apresentado interesse por parte da indústria farmacêutica, visto que muitas doenças estão relacionadas diretamente com distúrbios oxidativos.

\section{AGRADECIMENTOS}

À CAPES e CNPQ pela concessão das bolsas de estudo e financiamento do projeto e ao Museu Botânico Municipal da Prefeitura de Curitiba (MBM), pela identificação da espécie vegetal.

\section{REFERÊNCIAS}

ANDRADE, C.A. et al. Determinação do conteúdo fenólico e avaliação da atividade antioxidante de Acacia podalyriifolia A. Cunn. ex G. Don, Leguminosaemimosoideae. Revista Brasileira Farmacognosia, v.17, n.2, p.231-235, 2007

ARAÚJO, M.G.F.; CUNHA, W.R.; VENEZIANI, RCS. Estudo fitoquímico preliminar e bioensaio toxicológico frente a larvas de Artemia salina Leach. de extrato obtido de frutos de Solanum lycocarpum A. St.-Hill (Solanaceae). Revista de Ciências Farmacêuticas Básica e Aplicada, v.31, n.2, p.205-209, 2010.

BALESTRIN, L. et al. Contribuição ao estudo fitoquímico de Dorstenia multiformis Miquel (Moraceae) com abordagem em atividade antioxidante. Revista Brasileira de Farmacognosia, v.18, n.2, p.230-235, 2008

BARDÓN, A.E. Bioactive plants from Argentina and Bolivia. Fitoterapia, v.78, n.3, p.227-231, 2007.

BARROS, C.S.L. et al Biópsia hepática no diagnóstico da intoxicação por Senecio brasiliensis (Asteraceae) em bovinos. Pesquisa Veterinária. Brasileira, v.27, n.1, p.53-60, 2007.

BECHO, J.R.M, et al., Rutina - estrutura, metabolismo e potencial farmacológico. Revista Interdisciplinar de Estudos Experimentais, v.1, n.1, p.21 - 25, 2009.

BEDNARCZUK, V. O. et al. Testes in vitro e in vivo utilizados na triagem toxicológica de produtos naturais. Visão Acadêmica, v.11, n.2, p.44, 2010.

BOLZAN, A.A. et al Espécies de Senecio na medicina popular da américa latina e toxicidaade relacionada a sua utilização. Latin American Journal of Pharmacy, v.26, n.4, p.619-625, 2007.

CAMPOS, J.S.; FRASSON, A.P.Z. Avaliação da atividade antioxidante do extrato aquoso de Lafoensia pacari A. ST-HIL. em emulsão não-iônica. Revista de Ciências Farmacêuticas Básica e Aplicada, v.32, n.2, p.363368, 2011.

CARVALHO, J.L.S. et al. Termoestabilidade de processos extrativos de Nasturtium officinale R. Br., Brassicaceae por sistema Soxhlet modificado. Química Nova, v.32, n.4, p.1031-1035, 2009.

CERQUEIRA, F.M, et al., Antioxidantes dietéticos: controvérsias e perspectivas. Química Nova, v.30, n.2, p.441-449, 2007.

CHAVES, M.C.V. et al. Caracterização físico-química do suco da acerola. Revista de Biologia e Ciências da Terra, v.4, n.2, p.1-10, 2004.

CUADRA, P. et al. Biological activity of some Patagonian plants. Fitoterapia, v.76, n.7-8, p.718-721, 2005.

ELLEGARD, L.H. et al. Dietary plant sterols and cholesterol metabolism. Nutrition Reviews, v.65, n.1, p.39-45, 2007.

FRESCURA, V.D.S. et al. Compostos fenólicos em extratos de Rosmarinus officinalis I. sob cultivo fora do solo. Enciclopédia biosfera, v.9, n.17, p.755-761, 2013.

HARIPRASATH, L, et al., Gastroprotective effect of Senecio candicans DC on experimental ulcer models.

Rev. Bras. PI. Med., Campinas, v.17, n.4, supl. III, p.1031-1040, 2015. 
Journal Ethnopharmacology, v.140, n.1, p.145-150, 2012.

HIROTA, B.C.K. et al. C-glycosyl flavones and a comparative study of the antioxidant, hemolytic and toxic potential of Jatropha multifida leaves and bark. International Journal of Phytomedicine, v.4, n.1, p.1-5, 2012.

JÚNIOR, T.P.S.; PEREIRA, B. Exercício físico intenso como pró-oxidante: mecanismos de indução de estresse oxidativo e conseqüências. Revista Brasileira de Ciência e Movimento, v.16, n.3, p.1-26, 2008.

LIMA, F.O.; BEZERRA, A.S. Flavonoides e radicais livres. Disciplinarum Scientia, v.13, n.1, p.111-124, 2012.

LUCENA, R.B. et al. Doenças de bovinos no Sul do Brasil: 6.706 casos. Pesquisa Veterinária Brasileira, v.30, n.5, p.428-434, 2010.

LUTSENKO, E.A., et al., Vitamin C Prevents DNA Mutation Induced by Oxidative Stress. The Journal of Biological Chemistry, v.27, n.19, p.16.895-16.899, 2002.

MANSOUR, R.H.A.; SALEH, N.A.M. Flavonoids of three local Senecio species. National Research Centre, v.20, n.5, p.1180-1181, 1981.

MARTELLI, F.; NUNES, F.M.F. Radicais livres: em busca do equilíbrio. Ciência e Cultura, v.66, n.3, p.54-57, 2014.

MENSOR, L.L. et al. Screening of Brazilian plant extracts for antioxidant activity by the use of DPPH free radical method. Phytotherapy Research, v.15, n.2, p.127130, 2001.

MEYER, B.N. et al. Brine Shrimp: A convenient general biossays for active plant constituents. Planta Médica, v.45, n.1, p.31-34, 1982.

MEZACHE, N. et al. Fast counter current chromatography of n-butanolic fraction from Senecio giganteus (Asteraceae). Natural Product Communications, v.4, n.10, p. 1357-1362, 2009.

MORAIS, S.M. et al. Atividade antioxidante de óleos essenciais de espécies de Croton do nordeste do Brasil. Química Nova, v.29, n.5, p.907-910, 2006

MOREIRA, E.A. Marcha sistemática de análise em fitoquímica. Tribuna farmacêutica, v.47, n.1, p.1-19, 1979.
NGUTA, J.M. et al. Biological screening of kenya medicinal plants using Artemia salina L. (Artemiidae). Pharmacologyonline, v.2, p.458-78, 2011.

OLIVEIRA, D.S. et al. Vitamina C, carotenoides, fenólicos totais e atividade antioxidante de goiaba, manga e mamão procedentes da Ceasa do Estado de Minas Gerais. Acta Scientiarum. Health Sciences, v.33, n.1, p.89-98, 2011

PAULA, C.S. et al. Atividade antioxidante e toxicidade preliminar do extrato e frações obtidas das folhas e cascas do caule de Dasyphyllum tomentosum (Spreng.) Cabrera. Revista Brasileira de Plantas Medicinais, v.16, n.2, p.189-195, 2014.

PEREIRA, R.J.; CARDOSO, M.G. Metabólitos secundários vegetais e benefícios antioxidants. Journal of Biotechnology and Biodiversity, v.3, n.4, p.146-152, 2012.

PRIETO, P, et al., Spectrophotometric quantitation of antioxidant capacity through the formation of a Phosphomolybdenum Complex: specific application to the determination of vitamin E. Analytical Biochemistry, v.269, n.2, p.337-341, 1999.

RAMALHO, V.C.; JORGE, N. Antioxidantes utilizados em óleos, gorduras e alimentos gordurosos. Química Nova, v.29, n.4, p.755-760, 2006.

SCOTT, A.; KNOTT, M. Cluster-analysis method for grouping means in analysis of variance. Biometrics, v.30, n.3, p.507-512, 1974.

SANTI, M.M., et al., Determinação do perfil fitoquímico de extrato com atividade antioxidante da espécie medicinal Cordia verbenacea DC. por HPLC-DAD. Revista Brasileira de Plantas Medicinais, v.16, n.2, p.256-261, 2014.

SILVA, C.M, et al., Alcaloides pirrolizidínicos em espécies do gênero Senecio. Química Nova, v.29, n.5, p.10471053, 2006.

SOUSA, C.C.C., et al., Fenóis totais e atividade antioxidante de cinco plantas medicinais. Química Nova, v.30, n.2, p.351-355, 2007.

YOSHIDA, Y.; NIKKI, E. Antioxidant effects of phytosterol and its components. Journal of Nutricional Sciense and Vitaminology, v.49, n.4, p.277-280, 2003. 